\title{
Dynamic Movement Assessment and Functional Movement Screening for injury prediction: a systematic review
}

\author{
Dynamic Movement Assessment e Functional Movement Screen para predição de lesões: uma \\ revisão sistemática
}
Dynamic Movement Assessment y Functional Movement Screen para la predicción de la lesión: una revisión sistemática

Priscila dos Santos Bunn',2, Elirez Bezerra da Silva'

\begin{abstract}
Dynamic Movement Assessment ${ }^{\mathrm{TM}}\left(\mathrm{DMA}^{\mathrm{TM}}\right.$ ) and Functional Movement Screening ${ }^{\mathrm{TM}}$ (FMS ${ }^{\mathrm{TM}}$ ) are tools to predict the risk of musculoskeletal injuries in individuals who practice physical activities. This systematic review aimed to evaluate the association of $\mathrm{DMA}^{\mathrm{TM}}$ and $\mathrm{FMS}^{\mathrm{TM}}$ with the risk of musculoskeletal injuries, in different physical activities, categorizing by analysis. A research without language or time filters was carried out in November 2016 in MEDLINE, Google Scholar, SciELO, SCOPUS, SPORTDiscus, CINAHL and BVS databases using the keywords: "injury prediction", "injury risk", "sensitivity", "specificity", "functional movement screening", and "dynamic movement assessment". Prospective studies that analyzed the association between $\mathrm{DMA}^{\mathrm{TM}}$ and $\mathrm{FMS}^{\mathrm{TM}}$ with the risk of musculoskeletal injuries in physical activities were included. The data extracted from the studies were: participant's profile, sample size, injury's classification criteria, follow-up time, and the results presented, subdivided by the type of statistical analysis. The risk of bias was performed with Newcastle-Ottawa Scale for cohort studies. No study with DMA ${ }^{\mathrm{TM}}$ was found. A total of $20 \mathrm{FMS}^{\mathrm{TM}}$ studies analyzing one or more of the following indicators were included: diagnostic accuracy (PPV, NPV and AUC), odds ratios (OR) or relative risk (RR). FMS $^{\text {TM }}$ showed a sensitivity $=12$ to $99 \%$; specificity $=38$ to 97\%; PPV=25 to 91\%; NPV=28 to $85 \%$; $A \cup C=0.42$ to 0.68 ; $\mathrm{OR}=0.53$ to 54.5 ; and $\mathrm{RR}=0.16-5.44$. The $\mathrm{FMS}^{\text {TM }}$ has proven
\end{abstract}

to be a predictor of musculoskeletal injuries. However, due to methodological limitations, its indiscriminate usage should be avoided.

Keywords | Cumulative Trauma Disorders; Athletic Injuries; Movement.

RESUMO I A Dynamic Movement Assessment (DMA ${ }^{\mathrm{TM}}$ ) e o Functional Movement Screening (FMS ${ }^{\mathrm{TM}}$ ) são ferramentas utilizadas para classificar o risco de lesões musculoesqueléticas em indivíduos que praticam exercícios físicos. O objetivo da presente revisão sistemática foi avaliar a associação de DMA ${ }^{\text {TM }}$ e FMS ${ }^{\text {TM }}$ com o risco de lesões musculoesqueléticas em diferentes atividades físicas, categorizando por análise. Uma pesquisa sem filtros de idioma ou de tempo foi realizada em novembro de 2016 nas bases de dados MEDLINE, Google Scholar, SciELO, SCOPUS, SPORTDiscus, CINAHL e BVS, utilizando as palavras-chave: "predição de lesão", "risco de lesão", "sensibilidade", "especificidade", "functional movement screening" e "dynamic movement assessment". Foram incluídos estudos prospectivos que analisaram a associação entre $\mathrm{DMA}^{\mathrm{TM}}$ e $\mathrm{FMS}^{\mathrm{TM}}$ com o risco de lesões musculoesqueléticas em atividades físicas. Foram extraídos dos estudos: perfil dos participantes, tamanho da amostra, critérios de classificação da lesão, tempo de seguimento e os resultados apresentados, subdivididos pelo tipo de análise estatística. O risco de

Study conducted by the Graduate Program in Exercise and Sport Sciences at the Universidade do Estado do Rio de Janeiro (UERJ), Brazil. 'Programa de Pós-Graduação em Ciências do Exercício e do Esporte, Universidade do Estado do Rio de Janeiro (UERJ), Rio de Janeiro, Brazil.

${ }^{2}$ Centro de Educação Física Almirante Adalberto Nunes (Cefan), Marinha do Brasil, Rio de Janeiro (RJ), Brazil. 
viés foi realizado com a Escala Newcastle-Ottawa para estudos de coorte. Não foi encontrado nenhum estudo sobre a DMA ${ }^{\text {TM }}$. Foram incluídos 20 estudos, que analisaram um ou mais dos seguintes indicadores: acurácia diagnóstica (VPP, VPN e $A \cup C$ ), razão de chances (OR) ou risco relativo (RR). O FMS ${ }^{\mathrm{TM}}$ apresentou sensibilidade=12-99\%; especificidade=38-97\%; $V P P=25-91 \% ; \quad V P N=28-85 \% ; \quad A \cup C=0,42-0,68 ; \quad O R=0.53-54.5$; e $R R=0,16-5,44$. O FMS ${ }^{T M}$ apresentou-se como um método preditor de lesões musculoesqueléticas. Entretanto, devido às limitações metodológicas dos estudos, seu uso indiscriminado deve ser evitado.

Descritores | Transtornos Traumáticos Cumulativos; Traumatismos em Atletas; Movimento.

RESUMEN | Evaluación Dinámica del Movimiento ${ }^{\mathrm{TM}}\left(\mathrm{DM}^{\mathrm{TM}}\right)$ y Detección del Movimiento Funcional ${ }^{\mathrm{TM}}$ (FMS ${ }^{\mathrm{TM}}$ ) son herramientas para predecir el riesgo de lesiones musculoesqueléticas en individuos que practican actividades físicas. Esta revisión sistemática tuvo como objetivo evaluar la asociación de DMA $A^{T M} y$ FMS $^{\text {TM }}$ con el riesgo de lesiones musculoesqueléticas en diferentes actividades físicas y categorizarlas por análisis. En noviembre de 2016 se llevó a cabo una investigación sin filtros de idioma o de tiempo en las bases de datos MEDLINE, Google Scholar,
SciELO, SCOPUS, SPORTDiscus, CINAHL y BVS, utilizando las palabras clave: predicción de lesiones, riesgo de lesiones, sensibilidad, especificidad, detección del movimiento funcional y evaluación dinámica de movimientos. Se incluyeron estudios prospectivos que analizaron la asociación entre DMA ${ }^{\mathrm{TM}}$ y FMS ${ }^{\mathrm{TM}}$ con el riesgo de lesiones musculoesqueléticas en actividades físicas. Los datos extraídos de los estudios fueron: perfil del participante, tamaño de la muestra, criterios de clasificación de la lesión, tiempo de seguimiento y los resultados presentados, subdivididos por el tipo de análisis estadístico. El riesgo de sesgo se realizó con la Escala Newcastle-Ottawa para estudios de cohorte. No se encontró ningún estudio con DMA ${ }^{\mathrm{T}}$. Se incluyeron un total de 20 estudios FMS $^{\mathrm{TM}}$ que analizaron uno o más de los siguientes indicadores: precisión diagnóstica (VPP, VPN y $A B C$ ), odds ratios (OR) o riesgo relativo (RR). FMS $^{\text {TM }}$ mostró una sensibilidad = del 12 al 99\%; especificidad = del 38 al 97\%; VPP = del 25 al 91\%; VPN = del 28 al 85\%; $A B C=0,42$ a 0,68; $\mathrm{OR}=0,53$ a 54,5; y RR =0,16-5,44. El FMS ${ }^{\text {TM }}$ ha demostrado ser un predictor de lesiones musculoesqueléticas. Sin embargo, debido a limitaciones metodológicas, se debe evitar su uso indiscriminado.

Palabras clave | Transtornos de Traumas Acumulados; Traumatismos en Atletas; Movimiento.

\section{INTRODUCTION}

Musculoskeletal injuries are one of the main causes of morbidity in individuals who practice physical exercises ${ }^{1-3}$. Thus, several screening methods have been developed aiming at classifying the risk of injury. In this context, functional tests based on subjective evaluations have been increasingly performed ${ }^{4}$ to verify the movement patterns and dysfunctions associated with injuries of the trunk and lower limbs ${ }^{5,6}$. The subjectivity of these evaluations limits their reliability ${ }^{7}$. However, they are a low-cost alternative in large-scale evaluations and in case of absence of gold standards ${ }^{8}$.

To establish a risk classification tool for musculoskeletal injuries, Cook et al. ${ }^{9}$ developed the Functional Movement Screening ${ }^{\mathrm{TM}}\left(\mathrm{FMS}^{\mathrm{TM}}\right)$. This method classifies the risk of injury in the presence of abnormal movement patterns by performing seven tests/ movements ${ }^{9,10}$. Each test can be evaluated from zero to three points and assess the interactions of kinetic chain mobility and stability needed to perform fundamental movement patterns. The score ranges from 0 to 21 points.
Initial studies have shown that soccer players with scores of 14 or less in the total score have a higher risk of injury ${ }^{11}$. Thus, this method has been used in preseasons of several modalities of sports to modify movement patterns that can cause injuries ${ }^{10,12}$. However, the efficacy of FMS ${ }^{\mathrm{TM}}$ to predict injuries is controversial among authors $^{13-15}$, likely justified by the different demands among sports ${ }^{13}$.

Years later, Nessler \& Dunn developed the Dynamic Movement Assessment ${ }^{\mathrm{TM}}\left(\mathrm{DMA}^{\mathrm{TM}}\right)^{5}$. It consists of filming the individual performing six functional tests. The video analysis is performed using a twodimensional (2D) biomechanical analysis software. The $2 \mathrm{D}$ evaluation allows visualizing movement dysfunctions in the frontal plane, such as dynamic valgus ${ }^{8}$. Each of the six DMA ${ }^{\mathrm{TM}}$ tests is rated with a score ranging from zero (if the pain is related to the test) to three points. Each test has a major deviation and secondary deviations, which are observed. Failing to perform the test, the presence of three minor deviations, or a major deviation of greater magnitude characterizes a one-point score. The presence of two secondary 
deviations or a major deviation with intermediate magnitude promotes two points. Finally, individuals who perform the test without clinically important deviations are classified with three points ${ }^{5}$.

Due to the low cost and easy feasibility of the $\mathrm{FMS}^{\mathrm{TM}}$ and $\mathrm{DMA}^{\mathrm{TM}}$, their use to evaluate individuals who practice physical exercises in several groups is attractive. The main difference between the two methods is that $\mathrm{DMA}^{\mathrm{TM}}$ is based on functional tests with unilateral support (squatting and vertical jump), common in the sport gesture of several modalities ${ }^{5}$.

The fact that the incidence of injuries in people who practice sports ${ }^{16,17}$ or occupational physical exercises ${ }^{1,18,19}$ is high justifies this review. Getting to know a low-cost and easy-to-use test that measures fundamental movement dysfunctions, potentially predicting athletic injuries, may allow the development of preventive strategies that avoid the removal of functions involving physical exercises. Moreover, previous reviews evaluated only the diagnostic accuracy indicators of prospective studies of FMS ${ }^{\mathrm{TM}}$. Thus, the purpose of this systematic review is to evaluate the association of $\mathrm{DMA}^{\mathrm{TM}}$ and $\mathrm{FMS}^{\mathrm{TM}}$ with the risk of musculoskeletal injuries.

\section{METHODOLOGY}

This systematic review was registered in PROSPERO (CRD42017068014) and drafted based on the Preferred Reporting Items for Systematic Reviews and MetaAnalyses: The PRISMA Statement ${ }^{20}$.

\section{Inclusion criteria}

The studies included in this systematic review were prospective studies that used the $\mathrm{FMS}^{\mathrm{TM}}$ or $\mathrm{DMA}^{\mathrm{TM}}$ to classify the risk of musculoskeletal injuries in physical exercise practitioners of both sexes and without age limits. More detailed information about $\mathrm{FMS}^{\mathrm{TM}}$ and $\mathrm{DMA}^{\mathrm{TM}}$ are found in the studies of Cook et al., and Nessler \& Dunn, respectively, ${ }^{5,9}$.

\section{Search strategy}

A search was conducted in November 2016 in US National Library of Medicine (MEDLINE), Scientific Electronic Library Online (SciELO), Google Scholar, Virtual Health Library (VHL), CINAHL (EBSCOhost), SPORTDiscus and SCOPUS. The following keywords were used as descriptors of the Medical Subject Headings (MeSH): injury prediction, injury risk, functional movement screening, and dynamic movement assessment. The sentences used in this research were done with the Boolean operators AND (between the descriptors) and OR (between descriptor's synonyms). No date limits or language filters were applied.

\section{Data collection process}

The following data were extracted from the selected studies: profile of the participants, sample size, classification of musculoskeletal injuries, follow-up time and type of statistical analysis performed with its results.

\section{Bias risk analysis}

For bias risk analysis, the Newcastle-Ottawa scale was used ${ }^{21}$. The domains considered in this scale are: (1) selection (representativeness of the exposed cohort, selection of the unexposed cohort, evaluation of the exposure and confirmation that the result of interest was not present at the beginning of the study, (2) comparison of the cohort based on the study design or analysis (if results were adjusted for the main confounding factors and other variables) and (3) outcome (outcome assessment, sufficient follow-up time, and adequacy of cohort follow-up). Studies with less than five stars were classified as a "high risk of bias." In addition, studies were considered to have a "risk of uncertain bias" as they did not score in the "comparison" domain. The bias risk analysis was performed by only one evaluator.

\section{RESULTS}

\section{Flow diagram}

The total of studies per database and flow diagram of the studies are in Figure 1. Seven studies were manually located. None of the studies investigated the $\mathrm{DMA}^{\mathrm{TM}}$. Characteristics of the studies included are in Table 1. Statistical analysis and its result are shown in Table 2, and bias risk analysis are exposed in Table 3. 


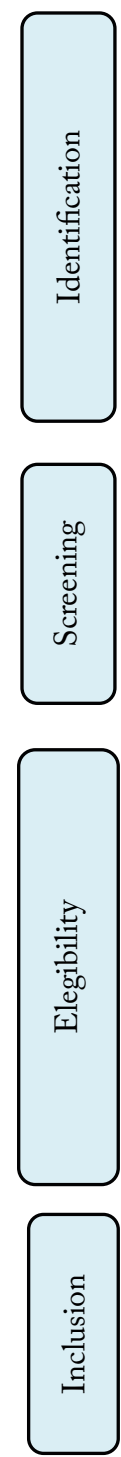

Studies retrieved from databases $(n=403)$

MEDLINE $(\mathrm{n}=180)$

Google Scholar $(n=38)$

$\operatorname{SciELO}(\mathrm{n}=0)$

$\operatorname{BVS}(n=0)$

CINAHL (EBSCOhost) $(\mathrm{n}=33)$

SPORTDiscuss $(\mathrm{n}=58)$

SCOPUS $(n=94)$

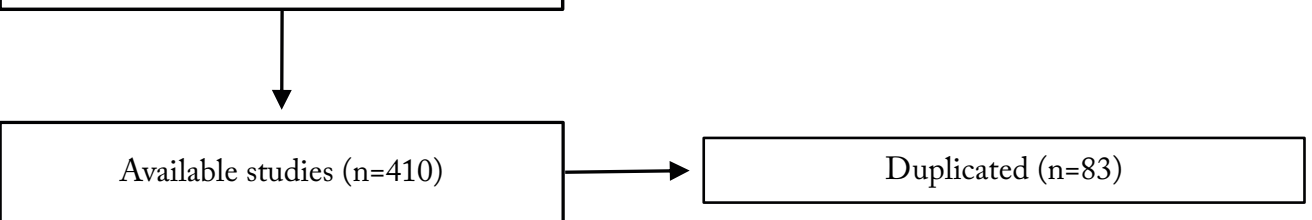

Available studies $(\mathrm{n}=410)$
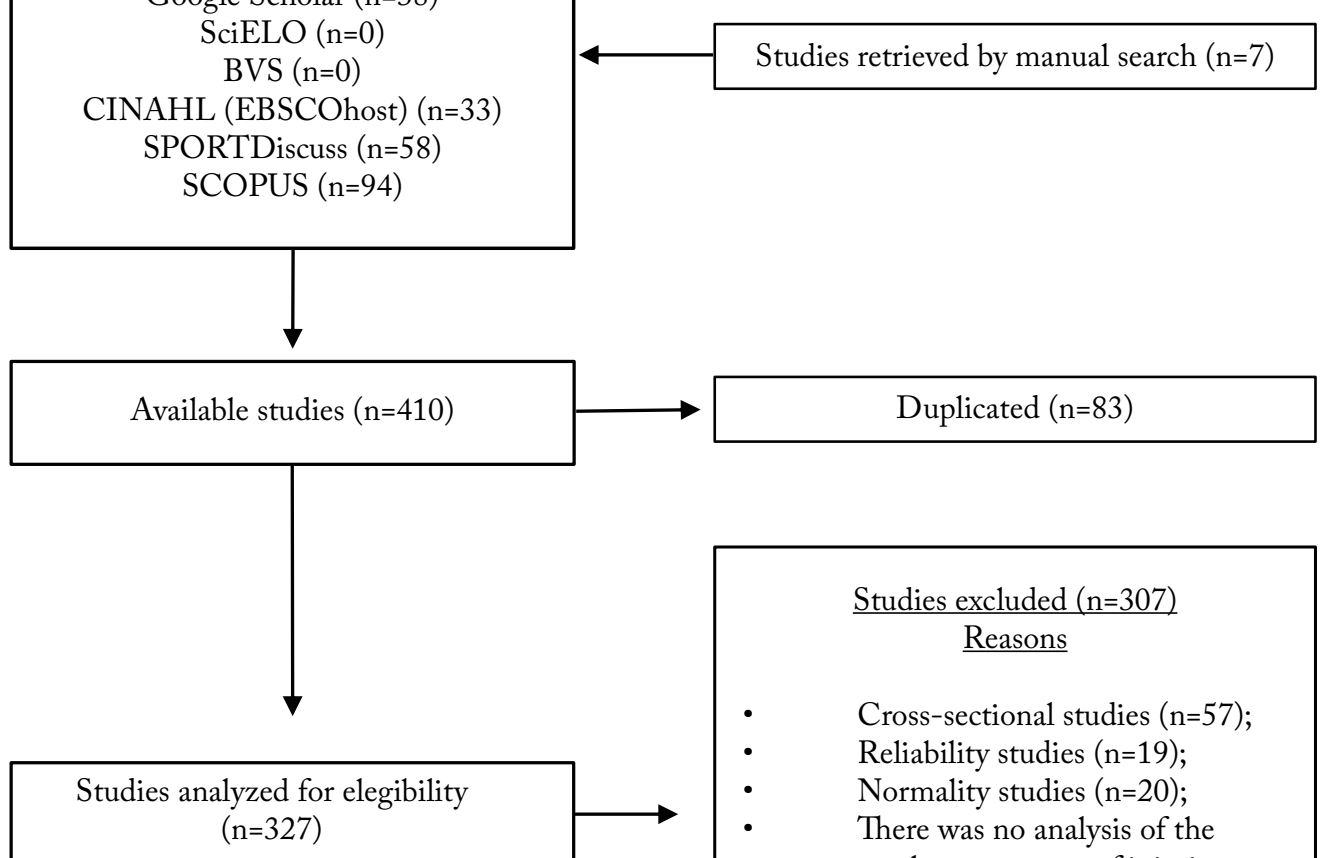

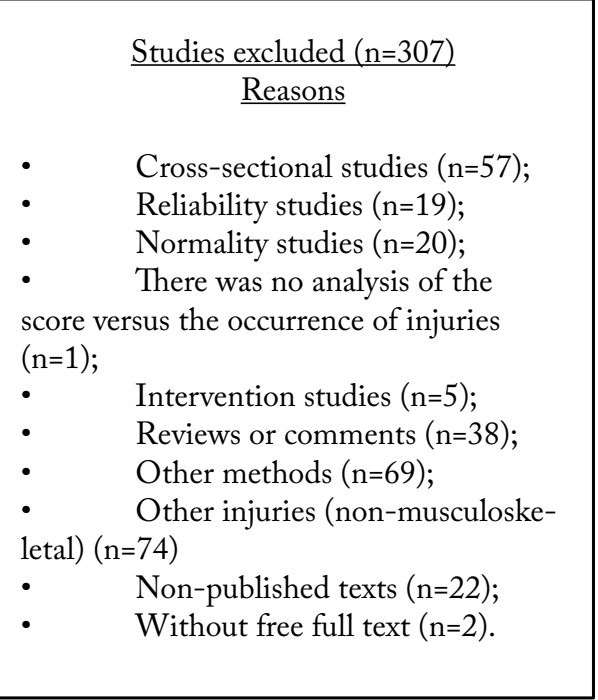

Figure 1. Flow diagram

Table 1. Characteristic of included studies

\begin{tabular}{|c|c|c|c|c|}
\hline Study & Participants & Sample & $\begin{array}{l}\text { Injury's } \\
\text { classification } \\
\text { criteria }\end{array}$ & Follow-up time \\
\hline Bushman et al., $2015^{22}$ & $\begin{array}{l}\text { Military } \\
\text { personnel }\end{array}$ & $\begin{array}{l}n=2476 \text { soldiers; } \\
\text { Age=18 }-57 \text { years old. }\end{array}$ & 1 & 6 months \\
\hline Lisman et al., $2013^{23}$ & $\begin{array}{l}\text { Military } \\
\text { personnel }\end{array}$ & $\begin{array}{l}n=447(S T) \text { and } n=427(L T) \text { marine officer's } \\
\text { students; } \\
\text { Age }=22.4 \pm 2.7 \text { years old. }\end{array}$ & I & $\begin{array}{c}6(n=427) \text { or } 10 \text { weeks }(n=447) \text {, } \\
\text { according with course (ST or } \\
\text { LT). }\end{array}$ \\
\hline Kodesh et al., $2015^{24}$ & $\begin{array}{l}\text { Military } \\
\text { personnel }\end{array}$ & $\begin{array}{l}n=158 \text { female soldiers from the Combat Fitness } \\
\text { Instructor Course (CFIC) - Israel Defense Forces } \\
\text { (IDF); } \\
\text { Age=19.0 (18.1 - 20.2) years old. }\end{array}$ & I & Three months \\
\hline Butler et al., $2013^{25}$ & $\begin{array}{l}\text { Military } \\
\text { personnel }\end{array}$ & $\begin{array}{l}n=108 \text { firefighter training course students; } \\
\text { Age=NR. }\end{array}$ & $\| I^{*}$ & 4 months \\
\hline McGill et al., $2015^{26}$ & $\begin{array}{l}\text { Military } \\
\text { personnel }\end{array}$ & $\begin{array}{l}\mathrm{n}=53 \text { men, elite police department; } \\
\text { Age }=37.9 \pm 5 \text { years old. }\end{array}$ & I, II & 5 years \\
\hline O'Connor et al., $2011^{27}$ & $\begin{array}{l}\text { Military } \\
\text { personnel }\end{array}$ & $\begin{array}{l}n=874 \text { marine officer's students; } \\
\text { Age }=18-30 \text { years old. }\end{array}$ & ।, III"* & $\begin{array}{c}6(n=427) \text { or } 10 \text { weeks }(n=447) \text {, } \\
\text { according with course (ST or } \\
L T) \text {. }\end{array}$ \\
\hline
\end{tabular}


Table 1. Continuation

\begin{tabular}{|c|c|c|c|c|}
\hline Study & Participants & Sample & $\begin{array}{l}\text { Injury's } \\
\text { classification } \\
\text { criteria }\end{array}$ & Follow-up time \\
\hline Warren et al., $2015^{28}$ & Athletes & $\begin{array}{l}n=167 \text { college athletes; } \\
\text { Age }=18-24 \text { years old. }\end{array}$ & । & One Season \\
\hline Kiesel et al., 2007"1 & Athletes & $\begin{array}{l}\mathrm{n}=46 \text { football players; } \\
\text { Age=NR. }\end{array}$ & $\| I^{*}$ & 4,5 months \\
\hline Tee et al., $2016^{29}$ & Athletes & $\begin{array}{l}\mathrm{n}=62 \text { rugby players; } \\
\text { Age }=\text { NR. }\end{array}$ & $\| I^{*}$ & 6 months \\
\hline Azzam et al., $2015^{30}$ & Athletes & $\begin{array}{l}\mathrm{n}=34 \text { basketball players; } \\
\text { Age=NR. }\end{array}$ & \|\|$^{\#}$ & One season \\
\hline Clay et al., $2016^{31}$ & Athletes & $\begin{array}{l}\mathrm{n}=45 \text { Division } \mathrm{I} \text { female collegiate rowers; } \\
\text { Age=at least } 18 \text { years of age; }\end{array}$ & III & One season \\
\hline Bardenett et al., $2015^{32}$ & Athletes & $\begin{array}{l}n=185 \text { ( } 97 \text { women, } 88 \text { men) high school athletes - } \\
\text { several modalities; } \\
\text { Age }=13 \text { - } 18 \text { years old. }\end{array}$ & II, III & One-quarter (autumn) \\
\hline Hammes et al., $2016^{33}$ & Athletes & $\begin{array}{l}n=238 \text { football players }>32 \text { years old; } \\
\text { Age }=44 \pm 7 \text { years old. }\end{array}$ & ।, III & 9 months \\
\hline Kiesel et al., $2014^{34}$ & Athletes & $\begin{array}{l}\mathrm{n}=238 \text { football players; } \\
\text { Age=NR. }\end{array}$ & ।, III & One preseason \\
\hline Chorba et al., $2010^{35}$ & Athletes & $\begin{array}{l}n=38 \text { collegiate athletes, women - several } \\
\text { modalities; } \\
\text { Age }=18-26 \text { years old. }\end{array}$ & I, II & One season \\
\hline Dossa et al., 201436 & Athletes & $\begin{array}{l}\mathrm{n}=20 \text { hockey players; } \\
\text { Age }=16-20 \text { years old. }\end{array}$ & $\|\|$, & $\begin{array}{l}\text { One season } \\
(2013-2014)\end{array}$ \\
\hline Weise et al., $2014^{37}$ & Athletes & $\begin{array}{l}\mathrm{N}=144 \text { collegiate athletes - football; } \\
\mathrm{Age}=18.9 \pm 1.3 \text { years old. }\end{array}$ & I, II, III & One season \\
\hline Garrison et al., $2015^{38}$ & Athletes & $\begin{array}{l}n=168 \text { collegiate athletes }- \text { several modalities } \\
\text { Age }=17-22 \text { years old. }\end{array}$ & I, II, III & One season \\
\hline Mokha et al, $2016^{39}$ & Athletes & $\begin{array}{l}\mathrm{n}=84(20 \text { men }) \text { - several modalities; } \\
\text { Age }=20.4 \pm 1.3 \text { years old (men) and } 19.1 \pm 1.2 \\
\text { (women). }\end{array}$ & I, II, III & One season \\
\hline Martin et al., $2016^{40}$ & Athletes & $\begin{array}{l}n=27 \text { adolescent Cricket Pace Bowlers } \\
\text { Age }=13-18 \text { years old. }\end{array}$ & I, II, III & One season \\
\hline
\end{tabular}

$\mathrm{N}=$ sample size; NR=Not reported; TI=traumatic injuries; NTI=nontraumatic injuries; $A l=a l l$ injuries; ICD=International Code of Diseases; $S T=$ short term course; $L T=I$ ong term course. Criteria used to define the injuries: I) the diagnosis of the injuries was performed by a healthcare professional; II) The registered injuries were related to the training or competition; and III) Only the musculoskeletal injuries with time-loss longer than 24 hours were considered lesions; $1 \|^{\prime \prime}=$ time-loss longer than 3 weeks; $\| I^{\prime \prime}=$ a severe injury promotes withdrawal from the training program; $\| I^{\sharp}=$ time-loss longer than 7 days.

Table 2. Statistical analysis

\begin{tabular}{|c|c|c|}
\hline $\begin{array}{l}\text { Statistical } \\
\text { analysis }\end{array}$ & Author & Results \\
\hline \multirow{11}{*}{$\begin{array}{l}\text { Diagnostic } \\
\text { accuracy }\end{array}$} & Bardenett et al., $2015^{32}$ & $A \cup C=0.49 ; I G$ versus NIG $(P=0.95) ; s=0.56 ; e=0,38,+L R=0.91 ;-L R=1.14$ \\
\hline & Bushman et al., $2015^{22}$ & $\begin{array}{l}\text { FMS (cutoff=14): NTI: s=37\%; e=81\%; PPV=43\%; NPV=77\% AUC: } 61 \% \text {; } \\
\text { TI: s=28\%; e=77\%; PPV =19\%; NPV=85\% AUC: } 54 \% ; \\
\text { Al: s=33\%; e=82\% VPP=52\%; VPN=68\% AUC: } 60 \%\end{array}$ \\
\hline & Garrison et al., $2015^{38}$ & $\begin{array}{l}\text { FMS (cutoff }=14): s=0.67, e=0.73,+L R=2.51 ;-L R=0.45 \\
\text { FMS (cutoff }=14 \text { ) + past injuries: } s=0.65, e=0.89,+L R=5.88 ;-L R=0.39\end{array}$ \\
\hline & Hammes et al., $2016^{33}$ & FMS (cutoff=14): AUC (any injury)=0.56; $\mathrm{Cl} 95 \%=0.47-0.64 ; \mathrm{P}=0.17 ; \mathrm{A} \cup \mathrm{C}(\mathrm{NTI})=0.55 ; \mathrm{Cl} 95 \%=0.46-0.64, \mathrm{P}=0.30)$ \\
\hline & Kiesel et al., 2007"11 & $\begin{array}{l}\text { FMS (cutoff=14): } s=0.54(\mathrm{Cl} 95 \%=0.34-0.68) ; e=0.91(\mathrm{Cl} 95 \%=0.83-0.96),+L R=5.92(\mathrm{Cl} 95 \%=1.97-18.37) \text {, } \\
-L R=0.51(\mathrm{Cl} 95 \%=0.34-0.79)\end{array}$ \\
\hline & Kiesel et al., $2014^{34}$ & $\begin{array}{l}\text { FMS (cutoff=14): } s=0.26(\mathrm{Cl} 95 \%=0.18-0.36), e=0.87(\mathrm{Cl} 95 \%=0.84-0.90 \\
\text { FMS (cutoff=14)+asymmetry: } \mathrm{e}=0.87(\mathrm{Cl} 95 \% 0.84-0.90)\end{array}$ \\
\hline & Chorba et al., $2010^{35}$ & $\begin{array}{l}\text { FMS (cutoff=14): } \mathrm{s}=0.579(\mathrm{Cl} 95 \%=0.335-0.798) ; e=0.737(\mathrm{Cl} 95 \%=0.488-0.909) ;+\mathrm{LR}=2.200 \\
(\mathrm{Cl} 95 \%=0.945-5.119)\end{array}$ \\
\hline & Mokha et al, $2016^{39}$ & FMS (cutoff=14): $s=26,3 \% ; e=58.7 \%$ \\
\hline & Dossa et al., $2014^{36}$ & $\begin{array}{l}\mathrm{S}=0.5(\mathrm{Cl} 95 \%=0.189-0.811) ; \mathrm{e}=0.7(\mathrm{Cl} 95 \%=0.348-0.930) ;+\mathrm{LR}=1.67(\mathrm{Cl} 95 \%=0.54-5.17) ;-\mathrm{LR}=0.71 \\
(\mathrm{Cl} 95 \%=0.34-1.50) ; \mathrm{PPV}=62.50 \%(\mathrm{Cl} 95 \%=0.25-0.91) ; \mathrm{NPV}=58.33 \%(\mathrm{Cl} 95 \%=0.28-0.85)\end{array}$ \\
\hline & McGill et al., $2015^{26}$ & $s=0.28$ (low back pain) e 0.42 (Al); e=0.76 (low back pain) and 0.47 (Al); P=NR \\
\hline & O'Connor et al., $2011^{27}$ & $\begin{array}{l}\mathrm{s}=0.452(\mathrm{Al}), 0.12 \text { (severe injury) and } 0.13(\mathrm{NTI}) \text {. } \\
\mathrm{e}=0.782(\mathrm{Al}), 0.939 \text { (severe injury) and } 0.901(\mathrm{NTI}) . A \cup C=0.58(\mathrm{Al}), 0.53 \text { (severe injury) and } 0.52(\mathrm{NTI}) \text {. }\end{array}$ \\
\hline
\end{tabular}


Table 2. Continuation

\begin{tabular}{|c|c|c|}
\hline $\begin{array}{l}\text { Statistical } \\
\text { analysis }\end{array}$ & Author & Results \\
\hline \multirow{4}{*}{$\begin{array}{l}\text { Diagnostic } \\
\text { accuracy }\end{array}$} & Weise et al., $2014^{37}$ & $\begin{array}{l}A \cup C=0.491(P=0,854), s=0.495 ; 1-e=0.429,+L R=1.154(P=0.819) ; \\
U L \text { injuries (FMS cutoff =17): } A \cup C=0.483(P=0.769) ; s=0.500 ; 1-e=0.464 ;+L R=1.078(P=0.765) \\
L L \text { injuries (FMS cutoff =17): } A \cup C=0.486(p=0.766) ; s=0.480 ; 1-e=0.464 ;+L R=1.035(P=0.762) \\
\text { NTI: } A \cup C=0.490(P=0.846) ; s=0.232 ; 1-e=0.216 ; R V P=1.075(P=0.843) ; \\
\text { Injuries with time-loss > } 10 \text { days: } A \cup C=0.422(P=0.194), s=0.996 ; e=0.974 ;+L R=0.992(P=0.187)\end{array}$ \\
\hline & Tee et al., $2016^{29}$ & $\mathrm{~s}=0.83(\mathrm{Cl} 95 \%=0.52-0.98) ; \mathrm{e}=0.46(\mathrm{Cl} 95 \%=0.35-0.48) ; \mathrm{A} \cup \mathrm{C}=0.68$ and $\mathrm{P}=0.049$ \\
\hline & Warren et al., $2015^{28}$ & $s=0.54 ; e=0.46 ; A \cup C=0.48$ \\
\hline & Kodesh et al., $2015^{24}$ & $s=0.42 ; e=0.63 ; A \cup C=0.51$ \\
\hline \multirow{12}{*}{$\begin{array}{l}\text { Odds } \\
\text { Ratios }\end{array}$} & Bushman et al., $2015^{22}$ & FMS (cutoff=14): OR=1.96 (P=0.01) \\
\hline & Garrison et al., $2015^{38}$ & FMS (cutoff=14): OR=5.71; Cl 95\%=2.73-11.51 \\
\hline & Kiesel et al., 2007"11 & FMS (cutoff=14): OR=11.67 (Cl 95\%=2.47-54.52) \\
\hline & Chorba et al., $2010^{35}$ & FMS (cutoff=14): OR=3.850 (Cl 95\%=0.980-15.13) \\
\hline & Mokha et al, $2016^{39}$ & $\begin{array}{l}\text { FMS (cutoff=14): OR=2.07 (P=0.15). } \\
\text { FMS (cutoff=14)+asymmetry: OR=5.27 (Cl 95\%=1.93-14.40; } P=0.001)\end{array}$ \\
\hline & O'Connor et al., $2011^{27}$ & $\begin{array}{l}\text { OR: } \mathrm{Al}(\mathrm{OR}=2.0 ;(\mathrm{Cl} 95 \%=1.3-3.1, \mathrm{P}=0.002), \mathrm{NTI}(\mathrm{OR}=1.4 ; \mathrm{Cl} 95 \%=0.71-2.6, \mathrm{P}=0.35) \text {; severe injuries } \\
(\mathrm{OR}=2.0 ; \mathrm{Cl} 95 \%=1.0-4.1 ; \mathrm{P}=0.05)\end{array}$ \\
\hline & Weise et al., $2014^{37}$ & $\begin{array}{l}\text { FMS (cutoff=17): Al-OR =1.425 (P=0.392); Cl 95\%=NR; UL injuries-OR=1.134 ( } P=0.793) ; \text { LL injuries-OR=1.113 } \\
(P=0.789) ; \\
\text { FMS (cutoff=18): NTI-OR=0.949 (P=0.926); FMS (cutoff=12): Injuries with "time-loss" >10 days: OR=2.154 } \\
(P=0.380)\end{array}$ \\
\hline & Lisman et al., $2013^{23}$ & 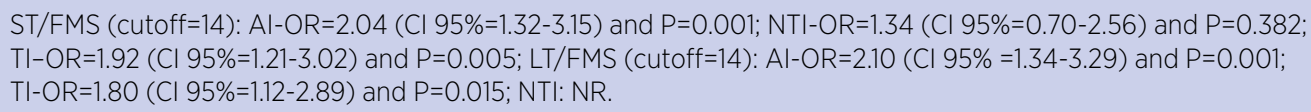 \\
\hline & Butler et al., $2013^{25}$ & OR: $1.21(\mathrm{Cl} 95 \%=1.01-1.42)$ \\
\hline & Tee et al., $2016^{29}$ & $\mathrm{OR}=4,3(\mathrm{Cl} 95 \%=0.9-21.0)$ \\
\hline & Warren et al., $2015^{28}$ & $\mathrm{OR}=1.01(\mathrm{Cl} 95 \%=0.53-1.91)$ \\
\hline & Kodesh et al., $2015^{41}$ & $\mathrm{OR}=0.98(\mathrm{Cl} 95 \%=0.87-1.10)$ \\
\hline \multirow{7}{*}{$\begin{array}{l}\text { Relative } \\
\text { Risk }\end{array}$} & Bushman et al., $2015^{22}$ & FMS (cutoff=14): RR=1.86 (NTI) and RR=1.49 (Al) $-\mathrm{P}=0.01)$ \\
\hline & Kiesel et al., $2014^{34}$ & FMS (cutoff=14): RR=1.87 (CI 95\%=1.20-2.96) \\
\hline & Mokha et al, $2016^{39}$ & $\begin{array}{l}\text { FMS (cutoff=14): RR=2.73 (Cl 95\% =1.36 - 5.44; } P=0.001) \\
\text { FMS (cutoff=18): RR=0.56 (Cl 95\%=0.34-0.93) }\end{array}$ \\
\hline & O'Connor et al., $2012^{27}$ & $\begin{array}{l}\text { ST - FMS (cutoff=14): RR }(\mathrm{Al})=1.91(\mathrm{Cl} 95 \%=1.21-3.01 ; \mathrm{P}<0.01) \\
\text { LT - FMS (cutoff=14): RR (AI)=1.65 (Cl 95\%=1.05-2.59; } p=0.03) \\
\text { ST+LT: RR }(A I)=1.5(P=0.003)\end{array}$ \\
\hline & Azzam et al., $2015^{30}$ & $\mathrm{RR}=0.86(\mathrm{Cl} 95 \%=0.42-1.76)$ \\
\hline & Martin et al., $2016^{40}$ & $\mathrm{RR}=0.59(\mathrm{Cl} 95 \%=0.16-2.20)$ \\
\hline & Kodesh et al., $2015^{41}$ & $\mathrm{RR}^{*}=1.49(\mathrm{Cl} 95 \%=0.998-2.23)$ \\
\hline
\end{tabular}

P=P-value; NR=Not reported; IG=injured group; NIG=non-injured group; CI 95\%=confidence interval to 95\%; s=sensibility; e=specificity; PPV=positive predictive value; NPV=negative predictive value; ROC: Receiver Operating Characteristic; AUC=area under the "receive operator curve"; TI=traumatic injuries; NTI=nontraumatic injuries; $A l=a l l$ injuries; FMS=Functional Movement Screen; +LR=Positive likelihood ratio; - $\mathrm{LR}=$ negative likelihood ratio; ICD=International Code of Diseases; UL=upper limbs; $L L=$ lower limbs; $S T=$ short term course; $L T=$ long term course; OR=odds ratios; RR=relative risk.

Table 3. Bias risk of studies that evaluated the association of FMS ${ }^{\mathrm{TM}}$ with the risk of musculoskeletal injuries with Newclastle-Ottawa Scale (NOS)

\begin{tabular}{|c|c|c|c|c|c|}
\hline Domain/study & Selection & Comparability & Outcome & Score & Risk \\
\hline Bushman et al. ${ }^{22}$ & $* * *$ & ** & * & $* * * * * *$ & Low \\
\hline Garrison et al. ${ }^{38}$ & $* *$ & * & $*$ & $* * * *$ & High \\
\hline Kiesel et al."1 & ** & - & $*$ & $* * *$ & High \\
\hline Lisman et al. ${ }^{23}$ & $* * * *$ & $* *$ & * & $* * * * * * *$ & Low \\
\hline Butler et al. ${ }^{25}$ & $* * * *$ & - & $* *$ & $* * * * * *$ & Uncertair \\
\hline Mokha et al. ${ }^{39}$ & $* * *$ & - & - & $* * *$ & High \\
\hline O'Connor et al..27 & $* * * *$ & ** & * & $* * * * * * *$ & Low \\
\hline Weise et al. ${ }^{37}$ & $* * *$ & - & * & $* * * *$ & High \\
\hline
\end{tabular}


Table 3. Continuation

\begin{tabular}{|c|c|c|c|c|c|}
\hline Domain/study & Selection & Comparability & Outcome & Score & Risk \\
\hline Bardenett et al..$^{32}$ & $* * *$ & - & ** & $* * * * *$ & Uncertain \\
\hline Dossa et al. ${ }^{36}$ & $* *$ & - & * & $* * *$ & High \\
\hline Hammes et al. ${ }^{33}$ & $* * *$ & ** & * & $* * * * * *$ & Low \\
\hline McGuill et al. ${ }^{26}$ & $* *$ & - & * & $* * *$ & High \\
\hline Azzam et al..$^{30}$ & $* *$ & - & ** & $* * * *$ & High \\
\hline Martin et al. ${ }^{40}$ & $* * *$ & - & ** & $* * * * *$ & Uncertain \\
\hline Warren et al. ${ }^{28}$ & $* * *$ & - & ** & $* * * * *$ & Uncertain \\
\hline Kodesh et al. ${ }^{24}$ & $* * *$ & - & * & $* * * *$ & High \\
\hline
\end{tabular}

Domains of Newcastle-Ottawa Scale (NOS): Selection (representativeness of the exposed cohort; selection of the nonexposed cohort; ascertainment of exposure and demonstration that the outcome of interest was not present at the beginning of the study); Comparability (principal factor and any additional factor); and Outcome (assessment of outcome; if the follow-up was long enough for producing outcomes; and adequacy of follow-up of cohorts).

\section{DISCUSSION}

This review aimed to evaluate the association between FMS $^{\mathrm{TM}}$ and $\mathrm{DMA}^{\mathrm{TM}}$ with the risk of musculoskeletal injuries. No studies with $\mathrm{DMA}^{\mathrm{TM}}$ were found, probably due to its recent development ${ }^{5}$. Based on the statistical analysis of most studies evaluated, $\mathrm{FMS}^{\mathrm{TM}}$ is associated with the risk of musculoskeletal injuries. Considering the cohort studies by Bushman et al. ${ }^{22}$ and O'Connor et al..$^{27}$, which had the lowest risk of bias, this association is strengthened (Table 3).

According to Table 2, the FMS ${ }^{\text {TM }}$ showed sensitivity values ranging from 26 to $68 \%$; specificity from 38 to $96 \%$; PPV from 19 to 91\%; NPV from 28 to $85 \%$; and AUC from 0.42 to 0.68 (Table 2). Therefore, it is noticeable that the indicators of diagnostic accuracy are divergent between the 12 studies. OR values also ranged from 0.53 to 11.67 , which corresponds, according to the literature, to absent and large effect sizes, respectively ${ }^{41}$. Only seven studies calculated the $\mathrm{RR}^{20,27,34,35,40,42,43}$, whose results were $\mathrm{RR}=1.86$ (overuse injuries) ${ }^{22}, \mathrm{RR}=1.49$ (traumatic injuries) ${ }^{22}$; and $R R=-0.5^{40}$ to $2.73^{39}$ (any injury). Thus, a low score in $\mathrm{FMS}^{\mathrm{TM}}$ is associated with higher injury risk, although this result is limited by the number of studies that calculate the RR and the high risk of bias in two of those studies ${ }^{27,34}$.

Considering the results of the studies with low risk of bias (Table 3), it is verified that FMS has a low sensitivity ${ }^{20,22,40}$, a good specificity ${ }^{20,33}$, and AUC values slightly above chance ${ }^{20,22}$. Three out of the four studies with low risk of bias used samples composed of soldiers ${ }^{20,33,40}$. These studies showed a higher score in the "selection" domain. This observation is a consequence of a greater representativeness of the samples in military courses, an adequate selection of the unexposed cohort (which is part of the same population) and the monitoring of the exhibition (based on the analysis of base records, such as military base records). At the same time, military groups are generally more homogeneous regarding various characteristics (age, level of fitness, volume of physical exercise, routine, etc.). Only six studies analyzed the influence of potential confounders ${ }^{20-22,33,40,41}$. Thus, all other studies have a "high" or "uncertain" risk of bias, once the influence of other risk factors on the result obtained was not reported. Most of the samples used had little representativeness, especially in studies with athletes. In addition, in many cases, attrition rates were neither quoted nor justified. In some cases, the absence of cases was not confirmed at the beginning of the studies, and it was not clear whether there was blinding of the participant and the professional responsible for the follow-up, which limits the interpretation of the results.

All the studies included in this systematic review had the same prospective design, in which the association between the score of $\mathrm{FMS}^{\mathrm{TM}}$ and the risk of injury were evaluated. Among 20 studies, 15 performed the diagnostic accuracy analysis, 12 calculated the OR and 7 the RR. The large variation between the results might have relation to several factors. First, samples consisted of athletes from different modalities or soldiers. In addition, the age of the individuals differed from one study to the other. Probably, $\mathrm{FMS}^{\mathrm{TM}}$ is not an appropriate assessment tool for every physical exercise practitioner. Second, the 
rating of injuries does not follow the same criteria in all studies. Some authors used the definition proposed by Hägglund et al. ${ }^{42}$, which defines a musculoskeletal injury when three criteria are related to injuries: association with athletic participation; necessity for health care; and time-loss with restrict participation for at least 24 hours. However, some authors included only severe injuries (with time-loss larger than three weeks) ${ }^{11}$ or any injury ${ }^{19,20,22,24,27,40,41}$. Third, statistical analysis based on indicators of diagnostic accuracy or simple calculation of OR limits the interpretation ${ }^{41}$. Diagnostic accuracy indicators are found in studies evaluating the validity of an index test compared with a reference standard ${ }^{43,44}$. In injury prediction studies, considering the occurrence of injuries as a reference pattern may limit the interpretation of the results, since a high-risk individual may not suffer an injury, especially if he/she is not exposed to the risk factor. The use of OR evaluates the chance of a highrisk individual to develop injuries. However, it does not consider the injury incidence ${ }^{41}$. Therefore, the most appropriate calculation is the relative risk.

This systematic review was the first to evaluate the association of $\mathrm{FMS}^{\mathrm{TM}}$ and $\mathrm{DMA}^{\mathrm{TM}}$, categorizing by type of statistical analysis performed. However, the small number of studies evaluating the RR of FMS ${ }^{\mathrm{TM}}$ and the absence of studies with DMA ${ }^{\mathrm{TM}}$ were limitations. In future studies, the control of some biases is recommended. Most of the studies did not perform pairing of variables such as gender, age and other variables of interest, such as sport modalities ${ }^{45}$. In this case, we suggest using logistic regression analysis. Another critical point was the lack of confirmation of case of absences in the baseline, as well the non-blinding of the evaluators responsible for monitoring the sample. In theory, they should not know whether the participant belonged to the group exposed to the risk factor. Finally, the development of studies about the association of DMA ${ }^{\mathrm{TM}}$ with the risk of musculoskeletal injuries is suggested, since no studies with this method were found, which uses movements present in several sport gestures with two-dimensional analysis ${ }^{5}$.

\section{CONCLUSION}

From the studies of this systematic review, the conclusion was that movement dysfunction, evaluated by $\mathrm{FMS}^{\mathrm{TM}}$, may be associated with the risk of injury in people who practice physical exercises. No studies evaluating the association between the $\mathrm{DMA}^{\mathrm{TM}}$ score and the risk of injury were found. It is recommended that future studies carry out greater control of selection, comparison and outcome biases, and perform a meta-analysis.

\section{ACKNOWLEDGMENTS}

We are grateful for the English language support provided by Lucas de Assis Borges.

\section{REFERENCES}

1. Taanila H, Suni JH, Kannus P, Pihlajamäki H, Ruohola J-P, Viskari J, et al. Risk factors of acute and overuse musculoskeletal injuries among young conscripts: a population-based cohort study. BMC Musculoskelet Disord. 2015;16(1):104. doi: 10.1186/s12891-015-0557-7

2. O'Brien J, Finch CF. The implementation of musculoskeletal injury-prevention exercise programmes in team ball sports: a systematic review employing the RE-AIM framework. Sport Med. 2014:1305-18. doi: 10.1007/s40279-014-0208-4

3. Taanila H, Suni J, Pihlajamäki H, Mattila VM, Ohrankämmen O, Vuorinen $\mathrm{P}$, et al. Aetiology and risk factors of musculoskeletal disorders in physically active conscripts: a follow-up study in the finnish defence forces. BMC Musculoskelet Disord. 2010;11:146. doi: 10.1186/1471-2474-11-146

4. McCunn R, Aus der Fünten K, Fullagar HHK, McKeown I, Meyer T. Reliability and association with injury of movement screens: a critical review. Sport Med. 2015;1-19. doi: 10.1007/s40279-015-0453-1

5. Nessler TD, Dunn EH. Dynamic movement assessment: prevent injury and enhance performance kindle edition. Publiwide, USA, 2014

6. Parkkari J, Taanila H, Suni J, Mattila VM, Ohrankämmen O, Vuorinen P, et al. Neuromuscular training with injury prevention counselling to decrease the risk of acute musculoskeletal injury in young men during military service: a populationbased, randomised study. BMC Med. 2011;9(1):35. doi: 10.1186/1741-7015-9-35

7. Munro A, Herrington L, Carolan M. Reliability of 2-dimensional video assessment of frontal-plane dynamic knee valgus during common athletic screening tasks. J Sport Rehabil. 2012;21:7-11.

8. Gwynne CR, Curran SA. Quantifying frontal plane knee motion during single limb squats: reliability and validity of 2-dimensional measures. Int J Sports Phys Ther. 2014;9(7):898-906.

9. Cook G, Burton L, Hoogenboom B. Pre-participation screening: the use of fundamental movements as an assessment of function - part 1. N Am J Sports Phys Ther. 2006;1(2):62-72.

10. Schneiders AG, Davidsson A, Hörman E, Sullivan SJ. Functional movement screen normative values in a young, active population. Int J Sports Phys Ther. 2011;6(2):75-82.

11. Kiesel K, Plisky PJ, Voight ML, Glaws KR, Juneau CM, Becker LC, et al. Can serious injury in professional football be predicted by a preseason functional movement screen? North Am J Sport Phys Ther. 2007;2(3):147-58. 
12. McCall A, Davison M, Andersen TE, Beasley I, Bizzini M, Dupont G, et al. Injury prevention strategies at the FIFA 2014 World Cup: perceptions and practices of the physicians from the 32 participating national teams. Br J Sports Med. 2015;49(9):603-8. doi: 10.1136/bjsports-2015-094747

13. Kraus K, Schültz E, Taylor WR, Doyscher R. Efficacy of the functional movement screen: a review. J Strength Cond Res. 2014;28(12):3571-84. doi: 10.1519/JSC.0000000000000556

14. Dorrel BS, Long T, Shaffer S, Myer GD. Evaluation of the functional movement screen as an injury prediction tool among active adult populations: a systematic review and metaanalysis. Sport Heal A Multidiscip Approach. 2015;7(6):532-7. doi: $10.1177 / 1941738115607445$

15. Krumrei K, Flanagan M, Bruner J, Durall C. The accuracy of the functional movement screen ${ }^{\mathrm{TM}}$ to identify individuals with an elevated risk of musculoskeletal injury. J Sport Rehabil. 2014;23(4):360-4. doi: 10.1123/jsr.2013-0027

16. McCall A, Carling C, Davison M, Nedelec M, Le Gall F, Berthoin $S$, et al. Injury risk factors, screening tests and preventative strategies: a systematic review of the evidence that underpins the perceptions and practices of 44 football (soccer) teams from various premier leagues. Br J Sports Med. 2015;49(9):583-9. doi: 10.1136/bjsports-2014-094104

17. Hoffman MD, Krishnan E. Health and exercise-related medical issues among 1,212 ultramarathon runners: baseline findings from the Ultrarunners Longitudinal TRAcking (ULTRA) Study. PLoS One. 2014;9(1):e83867. doi: 10.1371/journal.pone.0083867

18. Knapik JJ, Ang P, Reynolds K, Jones B. Physical fitness, age, and injury incidence in infantry soldiers. J Occup Med. 1993;35(6):598-603.

19. Knapik JJ, Graham B, Cobbs J, Thompson D, Steelman R, Jones $\mathrm{BH}$. A prospective investigation of injury incidence and injury risk factors among army recruits in military police training. BMC Musculoskelet Disord. 2013;14. doi: 10.1186/1471-2474-14-32

20. Moher D, Liberati A, Tetzlaff J, Altman DG, Grp P. Preferred reporting items for systematic reviews and meta-analyses: the PRISMA statement (Reprinted from Annals of Internal Medicine). Phys Ther. 2009;89(9):873-80. doi: 10.1371/journal.pmed.1000097

21. Wells GA, Shea B, Connell DO, Peterson J, Welch V, Losos M, et al. The Newcastle-Ottawa Scale (NOS) for assessing the quality of nonrandomised studies in meta-analyses [Internet]. 2000.

22. Bushman TT, Grier TL, Canham-Chervak M, Anderson MK, North WJ, Jones BH. The Functional Movement Screen and injury risk: association and predictive value in active men. Am J Sports Med. 2016;44(2):297-304. doi: 10.1177/0363546515614815

23. Lisman P, O'Connor FG, Deuster PA, Knapik JJ. Functional movement screen and aerobic fitness predict injuries in military training. Med Sci Sports Exerc. 2013;45(4):636-43. doi: 10.1249/MSS.0b013e31827a1c4c

24. Kodesh E, Shargal E, Kislev-Cohen R, Funk S, Dorfman L, Samuelly $\mathrm{G}$, et al. Examination of the effectiveness of predictors for musculoskeletal injuries in female soldiers. J Sport Sci Med. 2015:515-21.

25. Butler RJ, Contreras M, Burton LC, Plisky PJ, Goode A, Kiesel K. Modifiable risk factors predict injuries in firefighters during training academies. Work. 2013;46(1):11-7. doi: 10.3233/WOR-121545
26. McGill SM, Frost DM, Lam T, Finlay T, Darby K, Cannon J. Can fitness and movement quality prevent back injury in elite task force police officers? A 5-year longitudinal study. Ergonomics. 2015;139:1-8. doi: 10.1080/00140139.2015.1035760

27. O'Connor FG, Deuster PA, Davis J, Pappas CG, Knapik JJ. Functional movement screening: Predicting injuries in officer candidates. Med Sci Sports Exerc. 2011;43(12):2224-30. doi: 10.1249/MSS.0b013e318223522d

28. Warren M, Smith CA, Chimera NJ. Association of the Functional Movement Screen with injuries in division I athletes. J Sport Rehabil. 2015;24:163-70. doi: 10.1123/jsr.2013-0141.

29. Tee JC, Klingbiel JFG, Collins R, Lambert M, Coopoo Y. Preseason Functional Movement Screen component tests predict severe contact injuries in professional rugby union players. J Strength Cond Res. 2016; 30(11):3194-203. doi: 10.1519/JSC.0000000000001422

30. Azzam MG, Throckmorton TW, Smith RA, Graham D, Scholler J, Azar FM. The Functional Movement Screen as a predictor of injury in professional basketball players. Curr Orthop Pract. 2015;26(6):619-23. doi: 10.1097/BC0.0000000000000296

31. Clay H, Mansell J, Tierney R. Association between rowing injuries and the functional movement screen in female collegiate division I rowers. Int J Sports Phys Ther. 2016;11(3):345-9.

32. Bardenett SM, Micca JJ, DeNoyelles JT, Miller SD, Jenk DT, Brooks GS. Functional Movement Screen normative values and validity in high school athletes: can the FMS ${ }^{\mathrm{TM}}$ be used as a predictor of injury? Int J Sports Phys Ther. 2015;10(3):303-8.

33. Hammes D, Aus der Fünten K, Bizzini M, Meyer T. Injury prediction in veteran football players using the Functional Movement Screen $^{\text {TM }}$. J Sports Sci [Internet]. 2016;34(14):1371-9. Available from: http://www.ncbi.nlm.nih.gov/pubmed/26939907. doi: doi: 10.1080/02640414.2016.1152390

34. Kiesel KB, Butler RJ, Plisky PJ. Prediction of injury by limited and asymmetrical fundamental movement patterns in american football players. J Sport Rehabil. 2014;23(2):88-94. doi: 10.1123/jsr.2012-0130

35. Chorba RS, Chorba DJ, Bouillon LE, Overmyer CA, Landis JA. Use of a functional movement screening tool to determine injury risk in female collegiate athletes. N Am J Sports Phys Ther. 2010;5(2):47-54

36. Dossa K, Cashman G, Howitt S, West B, Murray N. Can injury in major junior hockey players be predicted by a pre-season functional movement screen - a prospective cohort study. J Can Chiropr Assoc. 2014;58(4):421-7.

37. Weise W, Boone J, Mattacola C, McKeon P, Lee T. Determination of the functional movement screen to predict musculoskeletal injury in intercollegiate athletics - PROQUEST. Athl Train Sport Healthc. 2014;6(4):161-9. doi: 10.3928/19425864-20140717-01

38. Garrison M, Westrick R, Johnson MR, Benenson J. Association between the functional movement screen and injury development in college athletes. Int J Sports Phys Ther. 2015;10(1):21-8.

39. Mokha M, Sprague PA, Gatens DR. Predicting musculoskeletal injury in national collegiate athletic association division II athletes from asymmetries and individual-test versus composite functional movement screen scores. J Athl Train. 2016;51(2). doi: 10.4085/1062-6050-51.2.07 
40. Martin AC, Olivier B, Benjamin N. The Functional Movement Screen in the prediction of injury in adolescent cricket pace bowlers: an observational study. J Sport Rehabil. 2017;26(5):386-95. doi: 10.1123/jsr.2016-0073

41. Chen $\mathrm{H}$, Cohen P, Chen S. How big is a big odds ratio? Interpreting the magnitudes of odds ratios in epidemiological studies. Commun Stat - Simul Comput. 2010;39(4):860-4. doi: 10.1080/03610911003650383

42. Hägglund M, Waldén M, Bahr R, Ekstrand J. Methods for epidemiological study of injuries to professional football players: developing the UEFA model. Br J Sports Med. 2005;39(6):340-6. doi: 10.1136/bjsm.2005.018267

43. Oliveira M, Gomes A, Toscano C. QUADAS and STARD: Evaluating the quality of diagnostic accuracy studies. Rev Saude Publica. 2011;45(2):416-22. doi: 10.1590/S0034-89102011000200021

44. Leeflang MMG, Deeks JJ, Gatsonis C, BossuytPMM. Systematic reviews of diagnostic test accuracy. Ann Intern Med. 2008;149(12):889-97. doi: 10.7326/0003-4819-149-12-200812160-00008

45. Margulis A, Pladevall M, Riera-guardia N, Varas-Iorenzo C, Hazell L, Berkman N, et al. Quality assessment of observational studies in a drug-safety systematic review, Comparison of two tools: The Newcastle-Ottawa scale and the RTI item bank. Clin Epidemiol. 2014;6:981-93. doi: 10.2147/CLEP.S66677

46. Frost DM, Beach TA, Callaghan JP MS. FMS Scores Change With Performers' Knowledge of the grading criteria-are general wholebody movement screens capturing "dysfunction"? J Strength Cond Res. 2015;29(11):3037-44. doi: 10.1097/JSC.0000000000000211 